\title{
Everything is on the head
}

\section{Thimios A. Mitsiadis*}

Institute of Oral Biology, Faculty of Medicine, University of Zurich, Zurich, Switzerland

${ }^{*}$ Correspondence: thimios.mitsiadis@zzm.uzh.ch

During the last 20 years, the field of craniofacial biology has benefited tremendously from the progress made in developmental biology and genomics. The huge quantity of data on numerous genes that are involved in developmental processes has considerably increased our comprehension of the organization and function of molecular networks that are used to build the head. New ideas and concepts have emerged that promote understanding the generation of craniofacial pathologies and imagining new therapeutic treatments. Gene therapy and stem cell research continue to develop rapidly, thereby providing novel information that broadens our scientific horizons. These important advancements led to the launch of the "Frontiers in Craniofacial Biology" journal. It is the goal of this new multidisciplinary journal to cover a broad spectrum of topics such as craniofacial evolution, development, genetics, pathology, molecular mechanisms in differentiation, mineralization and regeneration, stem cell applications, biotechnology, and tissue engineering.

Craniofacial (cranium, face, and jaws) development is a complex threedimensional process that involves the patterning, outgrowth, fusion, and molding of various heterogeneous tissues. The embryonic vertebrate face is initially composed of buds of mesenchyme encased in epithelium that surround the primitive oral cavity (stomodeum). Although similar in several vertebrate classes, these embryonic facial prominences give rise to markedly different postnatal forms. Recent data from different vertebrates support the idea that similar signals and processes are used in different species and thus what we learn in one model system is applicable to the next. Indeed, human genetic studies have identified specific gene mutations that affect facial patterning (Handrigan et al., 2007), genes that in many cases have been experimentally shown to be important for facial development in mouse or other model organisms.
The cells that contribute to the facial skeleton have their origins in cranial neural crest cells. These cells arise from the dorsal edges of the folded neural plate, through a series of interactions with the ectoderm (McCabe and Bronner-Fraser, 2009). The ability to form skeletogenic tissues is unique to the cranial neural crest cells that are divided into an anterior (or facial) domain and a posterior domain. Migrating facial neural crest cells and paraxial mesoderm combine to form the mesenchyme of the facial prominences. The mesoderm contributes the jaw musculature and the cranial base, while neural crest-derived mesenchyme forms the teeth, cartilage, intramembranous bone cells, and connective tissues of the face (Minoux and Rijli, 2010). Genetic manipulations in mice have begun to uncover the important role of epithelial-mesenchymal interactions that play in craniofacial development by targeting genes expressed in one tissue layer. It has been determined that different signaling molecules are used to pattern the different head regions, while transcription factors control facial prominence identities.

Genomics have considerably increased our understanding on cell plasticity, pathology, and regeneration, and, therefore, profoundly changed many paradigms used to define health and diseases. While in the past diseases were described only in histopathological and anatomical terms, today they are described also in terms of gene mutation. Gene therapy could open new horizons for the treatment of craniofacial malformations and diseases.

Tissue engineering is an area that has greatly profited from molecular biology. Tissue engineering techniques allow the replacement of damaged or lost tissues/ organs by new ones. Different approaches that combine biomaterials, biologic molecules, and cell transplantation techniques are used for the growth and/or regeneration of craniofacial tissues. These developments aim to regenerate craniofacial structures such as muscles, bones, cartilage, dentin, enamel, dental pulp, and periodontium and will have an obvious impact on craniofacial medicine and dentistry (Bluteau et al., 2008).

Stem cell research rejuvenated the field of craniofacial biology. The isolation of embryonic stem cells has allowed genetic manipulations that contributed to our thorough understanding of vertebrate embryonic development (Evans and Kaufman, 1981). Recent studies have shown that it is possible to reprogram human differentiated cells into pluripotent embryonic stem cells (called iPS) after expressing a cocktail of transcription factors (Takahashi and Yamanaka, 2006). These advances will certainly facilitate the development of in vitro models for many human diseases and provide researchers with new tools for regenerative purposes. A big part of stem cell research focuses on the identification of adult stem cell populations in the various tissues and organs of the human body. The mechanisms that underlie stem cell self-renewal and differentiation are largely derived from those used in development and pathology. The recent advances in stem cell biology contributed to the creation of a new medical discipline, regenerative medicine. Replacing defective or lost cells in patients with either stem cells or reprogrammed cells (iPS) is an attractive possibility to treat various pathologies. The possibility of autologous cell transplantation could remove the need for immunosuppression with the obvious resulting benefits. The use of cells naturally occurring in the injury site may minimize the risk of side effects. Stem cells must be expanded and differentiated into the appropriate tissue prior to grafting. However, the use of expanded cell populations needs to take into account the possibility of genetic and epigenetic instability. An important challenge for scientists working on this field is to master stem cell differentiation into a specific tissue of the craniofacial complex. Previous discoveries in the fields of craniofacial development and genetics will certainly contribute in achieving this goal. 
Despite the large amount of interest in stem cell research, not many clinical trials have been performed yet. Stem cell and material sciences have to define conditions for manufacturing consistent and reproducible products, which are quality controlled for safety and efficacy. "Frontiers in Craniofacial Biology" will promote and publish important findings on stem cell research linked to the craniofacial complex. Several of our editors who specialize in stem cell research will support this effort.

The above-mentioned progress opened the field of craniofacial research to other disciplines such as physic and mathematics. These interdisciplinary approaches have led to the development of new concepts, ideas and methods that, in turn, have boosted enormously the field. In addition, the rapid pace of technological progress in imaging sciences has helped advance various biological disciplines including craniofacial biology. Many questions concerning the mechanisms of head morphogenesis or differentiation have not yet been resolved but new sophisticated imaging tools, mathematics, bioinformatics, and genomics will help to elaborate concepts and create novel models, changing drastically the field of craniofacial research. However, it is crucial to control the basic biological tools and concepts before moving toward physics, mathematics, and imaging. The integration of these new approaches into established research program represents a major challenge and often a source of discouragement for some senior scientists working in the field of craniofacial biology. Young scientists who enter the field must not only master these new techniques but also must be prepared to adapt to meet these ever-changing challenges as the field progresses.

Entirely web-based scientific publishing is rapidly evolving. Websites are getting every day more modern with major redesign, new platforms, enhanced functionality, and navigation. Many scientists do not have the time to consult printed journals in libraries, and consequently more and more information is acquired exclusively via the web. Therefore, a major challenge for "Frontiers in Craniofacial Biology" is to keep its site modern, attractive, interesting, and highly visible for scientists and practitioners.

In closing, craniofacial biology is a field of broad interest and deserves a prominent place among other biological disciplines. Further progress in craniofacial and dental treatments depends upon continued active and vigorous research in our field. The increased information on craniofacial evolution, development, growth, homeostasis, pathology, and regeneration will translate basic research findings into daily practice techniques. I would like to take this opportunity to show my gratitude to the highly respected and experienced colleagues that accepted to be part of the editorial team. Their valuable expertise and active participation in the journal can guarantee the success of "Frontiers in Craniofacial Biology" by establishing exceptional publication standards and ethics in a period of growing competition from existing or newly created journals. We are looking very much forward to confronting and mastering these challenges.

\section{REFERENCES}

Bluteau, G., Luder, H. U., De Bari, C., and Mitsiadis, T. A. (2008). Stem cells for tooth engineering. Eur. Cell Mater. 16, 1-9.

Evans, M. J., and Kaufman, M. H. (1981). Establishment in culture of pluripotential cells from mouse embryos. Nature 292, 154-156.

Handrigan, G. R., Buchtová, M., and Richman, J. M. (2007). Gene discovery in craniofacial development and disease-cashing in your chips. Clin. Genet. 71, 109-119.

McCabe, K. L., and Bronner-Fraser, M. (2009). Molecular and tissue interactions governing induction of cranial ectodermal placodes. Dev. Biol. 332, 189-195.

Minoux, M., and Rijli, F. M. (2010). Molecular mechanisms of cranial neural crest cell migration and patterning in craniofacial development. Development 137, 2605-2621.

Takahashi, K., and Yamanaka, S. (2006). Induction of pluripotent stem cells from mouse embryonic and adult fibroblast cultures by defined factors. Cell 126, 663-676.

Received: 24 December 2010; accepted: 04 January 2011; published online: 21 January 2011.

Citation: Mitsiadis TA (2011) Everything is on the head. Front. Physio. 2:2. doi:10.3389/fphys.2011.00002

This article was submitted to Frontiers in Craniofacial Biology, a specialty of Frontiers in Physiology.

Copyright (C) 2011 Mitsiadis. This is an open-access article subject to an exclusive license agreement between the authors and Frontiers Media SA, which permits unrestricted use, distribution, and reproduction in any medium, provided the original authors and source are credited. 